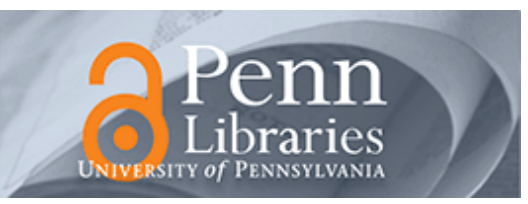

University of Pennsylvania

ScholarlyCommons

$1-1-2002$

\title{
An Idea for Thin Subwavelength Cavity Resonators Using Metamaterials With Negative Permittivity and Permeability
}

\author{
Nader Engheta \\ University of Pennsylvania, engheta@seas.upenn.edu
}

Follow this and additional works at: https://repository.upenn.edu/ese_papers

Part of the Electrical and Computer Engineering Commons

\section{Recommended Citation}

Nader Engheta, "An Idea for Thin Subwavelength Cavity Resonators Using Metamaterials With Negative Permittivity and Permeability", . January 2002.

Copyright 2002 IEEE. Reprinted from IEEE Antennas and Wireless Propagation Letters, Volume 1, Issue 1, 2002, pages 10-13.

Publisher URL: http://ieeexplore.ieee.org/xpl/tocresult.jsp?isNumber=21952\&puNumber=7727

This material is posted here with permission of the IEEE. Such permission of the IEEE does not in any way imply IEEE endorsement of any of the University of Pennsylvania's products or services. Internal or personal use of this material is permitted. However, permission to reprint/republish this material for advertising or promotional purposes or for creating new collective works for resale or redistribution must be obtained from the IEEE by writing to pubs-permissions@ieee.org. By choosing to view this document, you agree to all provisions of the copyright laws protecting it.

This paper is posted at ScholarlyCommons. https://repository.upenn.edu/ese_papers/12

For more information, please contact repository@pobox.upenn.edu. 


\title{
An Idea for Thin Subwavelength Cavity Resonators Using Metamaterials With Negative Permittivity and Permeability
}

\author{
Abstract \\ In this letter, we present and analyze theoretically some ideas for thin one-dimensional (1-D) cavity \\ resonators in which a combination of a conventional dielectric material and a metamaterial possessing \\ negative permittivity and permeability has been inserted. In this analysis, it is shown that a slab of \\ metamaterial with negative permittivity and permeability can act as a phase compensator/conjugator \\ and, thus, by combining such a slab with another slab made of a conventional dielectric material one can, \\ in principle, have a 1-D cavity resonator whose dispersion relation may not depend on the sum of \\ thicknesses of the interior materials filling this cavity, but instead it depends on the ratio of these \\ thicknesses. In other words, one can, in principle, conceptualize a 1-D cavity resonator with the total \\ thickness far less than the conventional $\lambda / 2$. Mathematical steps and physical intuitions relevant to this \\ problem are presented.
}

\section{Keywords}

Cavity resonator, metamaterials, negative index of refraction, negative permeability, negative permittivity, phase compensator, phase conjugation.

\section{Disciplines}

Electrical and Computer Engineering

\section{Comments}

Copyright 2002 IEEE. Reprinted from IEEE Antennas and Wireless Propagation Letters, Volume 1, Issue 1, 2002, pages 10-13.

Publisher URL: http://ieeexplore.ieee.org/xpl/tocresult.jsp?isNumber=21952\&puNumber=7727

This material is posted here with permission of the IEEE. Such permission of the IEEE does not in any way imply IEEE endorsement of any of the University of Pennsylvania's products or services. Internal or personal use of this material is permitted. However, permission to reprint/republish this material for advertising or promotional purposes or for creating new collective works for resale or redistribution must be obtained from the IEEE by writing to pubs-permissions@ieee.org. By choosing to view this document, you agree to all provisions of the copyright laws protecting it. 


\title{
An Idea for Thin Subwavelength Cavity Resonators Using Metamaterials With Negative Permittivity and Permeability
}

\author{
Nader Engheta, Fellow, IEEE
}

\begin{abstract}
In this letter, we present and analyze theoretically some ideas for thin one-dimensional (1-D) cavity resonators in which a combination of a conventional dielectric material and a metamaterial possessing negative permittivity and permeability has been inserted. In this analysis, it is shown that a slab of metamaterial with negative permittivity and permeability can act as a phase compensator/conjugator and, thus, by combining such a slab with another slab made of a conventional dielectric material one can, in principle, have a 1-D cavity resonator whose dispersion relation may not depend on the sum of thicknesses of the interior materials filling this cavity, but instead it depends on the ratio of these thicknesses. In other words, one can, in principle, conceptualize a 1-D cavity resonator with the total thickness far less than the conventional $\lambda / 2$. Mathematical steps and physical intuitions relevant to this problem are presented.
\end{abstract}

Index Terms-Cavity resonator, metamaterials, negative index of refraction, negative permeability, negative permittivity, phase compensator, phase conjugation.

\section{INTRODUCTION}

$\mathbf{I}^{N}$ $\mathrm{N}$ the past several decades, the electromagnetic (EM) properties of complex media have been the subject of research study for many research groups [1]-[16]. Several types of EM complex media such as chiral materials, omega media, bianisotropic media, local, and nonlocal media to name a few, have been studied. Recently, the idea of composite materials in which both permittivity and permeability possess negative values at certain frequencies has gained considerable attention [17]-[21]. In 1967, Veselago theoretically investigated plane wave propagation in a material whose permittivity and permeability were assumed to be simultaneously negative [22]. His theoretical study showed that for a monochromatic uniform plane wave in such a medium, the direction of the Poynting vector is antiparallel with the direction of phase velocity, contrary to the case of plane wave propagation in conventional simple media. Recently, Smith et al. constructed such a composite medium for the microwave regime and demonstrated experimentally the presence of anomalous refraction in this medium [17], [18], [20], [21]. It is also worth noting that previous theoretical study of EM wave interaction with omega media reveals the possibility of having negative

Manuscript received December 20, 2001; revised February 11, 2002. This work was presented in part at the International Conference on Electromagnetics in Advanced Applications (ICEAA), Torino, Italy, September 10-14, 2001.

The author is with the Department of Electrical Engineering, University of Pennsylvania, Philadelphia, PA 19104 USA (e-mail: engheta@ee.upenn.edu).

Digital Object Identifier 10.1109/LAWP.2002.802576 permittivity and permeability in omega media for a certain range of frequencies [23]. For metamaterials with negative permittivity and permeability, several names and terminologies have been suggested such as "left-handed" media [17]-[22], media with negative refractive index [17]-[22] "backward media" (BW media) [24], "double negative metamaterials" [25], [26], to name a few. The anomalous refraction at the boundary of such media and the fact that for a plane wave the direction of the Poynting vector is antiparallel with the direction of phase velocity, provide us with features that can be advantageous in design of novel devices and components. Recently, we introduced and presented in a symposium [27] one of our ideas for a compact cavity resonator. Here in this letter, we describe the details of this idea and the mathematical steps behind our analysis.

\section{A. Metamaterials With Negative $\varepsilon$ and $\mu$ as Phase Compensators/Conjugators}

When a lossless metamaterial possesses negative real permittivity and permeability at certain frequencies, the index of refraction in such a medium attains real values. So as theoretically predicted by Veselago, the EM wave can propagate in such a medium [22]. However, for a monochromatic uniform plane wave in such a medium the phase velocity is in the opposite direction of the Poynting vector.

Consider a slab of conventional lossless material with real permittivity $\varepsilon_{1}>0$, real permeability $\mu_{1}>0$, and the index of refraction $n_{1} \equiv \sqrt{\left(\varepsilon_{1} \mu_{1}\right) /\left(\varepsilon_{o} \mu_{o}\right)}$, where $\varepsilon_{o}$ and $\mu_{o}$ are the permittivity and permeability of the free space. Here, $n_{1}$ is taken to be a positive real quantity. The slab is infinitely extent in the $x-y$ plane and has the thickness $d_{1}$ along the $z$ axis. We temporarily assume that the intrinsic impedance of the dielectric material $\eta_{1}=\sqrt{\mu_{1} / \varepsilon_{1}}$ is the same as that of the outside region $\eta_{o}=\sqrt{\mu_{o} / \varepsilon_{o}}$, i.e., $\eta_{1}=\eta_{o}$, but its refractive index is different from that of outside, i.e., $n_{1} \neq n_{o}$. (We will soon remove the first part of this assumption.) Let us assume that a monochromatic uniform plane wave is normally incident on this slab. The wave propagates through the slab without any reflection (because for now we are still assuming $\eta_{1}=\eta_{o}$ ). As this wave traverses this slab, the phase at the end of the slab is obviously different from the phase at the beginning of the slab by the amount $n_{1} k_{o} d_{1}$, where $k_{o} \equiv \omega \sqrt{\varepsilon_{o} \mu_{o}}$. Now, consider a slab of a lossless metamaterial with negative real permittivity and permeability, i.e., $\varepsilon_{2}<0$ and $\mu_{2}<0$ at certain frequencies. For this slab, the index of refraction is a real quantity denoted by 


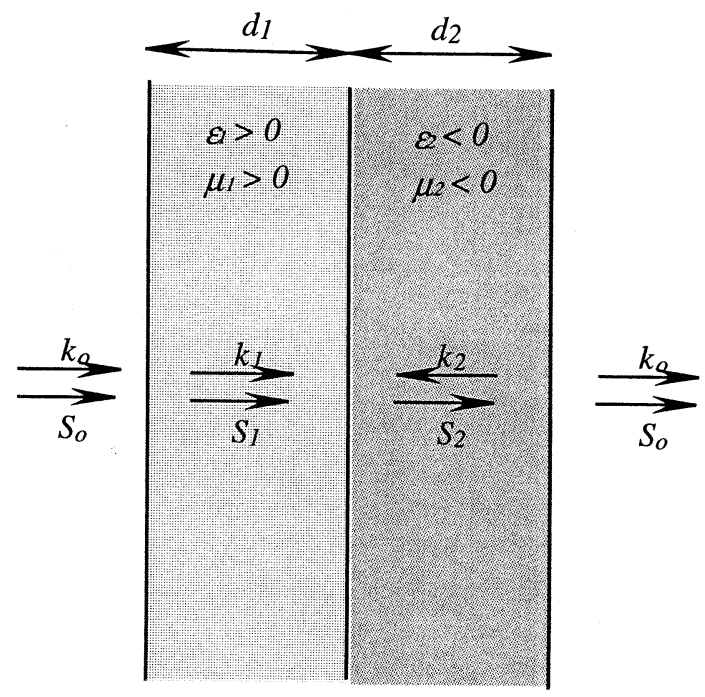

Fig. 1. A two-layer structure in which the left layer is assumed to be a conventional lossless dielectric material with $\varepsilon_{1}>0$ and $\mu_{1}>0$ and the right layer is taken to be a lossless metamaterial with negative permittivity and permeability. In the first layer, the direction of Poynting vector $\left(S_{1}\right)$ is parallel with the direction of phase velocity or wave vector $\left(k_{1}\right)$, whereas in the second layer, these two directions are antiparallel. With proper choice of ratio of $d_{1}$ and $d_{2}$, one can have the phase of the wave at the left (entrance) interface to be the same as the phase at the right (exit) interface, essentially with no constraint on the total thickness of the structure.

$n_{2} \equiv \sqrt{\left(\varepsilon_{2} \mu_{2}\right) /\left(\varepsilon_{o} \mu_{o}\right)}$. It is important to note that here we do not need to specify any sign for the operation of the square root appearing in the expression of $n_{2}$. We only need to state that $n_{2}$ is a real quantity for the lossless metamaterial with $\varepsilon_{2}<0$ and $\mu_{2}<0$ for a given frequency. (Here, $n_{2}$ can, for example, be taken to be a positive real quantity.) This slab is also infinitely extent in the $x-y$ plane, but has a thickness of $d_{2}$ in the $z$ direction. For now, we again assume that the intrinsic impedance of this metamaterial $\eta_{2}=\sqrt{\mu_{2} / \varepsilon_{2}}$ is also the same as that of outside region, i.e., $\eta_{2}=\eta_{o}$. We put this slab right next to the first slab (Fig. 1). As the plane wave exits the first slab, it enters the slab of metamaterial and finally it leaves this second slab. The direction of power flow (i.e., the Poynting vector) in the first slab should be the same as that in the second one, because the power of the incident wave enters the first slab (without any reflection at the first interface), traverses the first slab, exits the second interface, enters the second slab and traverses it, and finally leaves the second slab. In the first slab, the direction of the Poynting vector is parallel with the direction of phase velocity; however, in the second slab these two vectors are antiparallel (see Fig. 1). Therefore, the wave vector $k_{2}\left(\equiv n_{2} k_{o}\right)$ is in the opposite direction of the wave vector $k_{1}\left(\equiv n_{1} k_{o}\right)$. As a result, the phase at the end of the second slab is different from the phase at the beginning of it by the amount $-n_{2} k_{o} d_{2}$. (As was mentioned above, $n_{2}$ here is taken to be positive.) So the total phase difference between the front and back faces of this two-layer structure is $n_{1} k_{o} d_{1}-n_{2} k_{o} d_{2}$. Therefore, whatever phase difference is developed by traversing the first slab, it can be decreased and even cancelled by traversing the second slab. If the ratio of $d_{1}$ and $d_{2}$ is chosen to be $d_{1} / d_{2}=n_{2} / n_{1}$, then the total phase difference between the front and back faces of this two-layer structure becomes zero. (The total phase difference is not $2 \pi, 4 \pi$, or $6 \pi$.

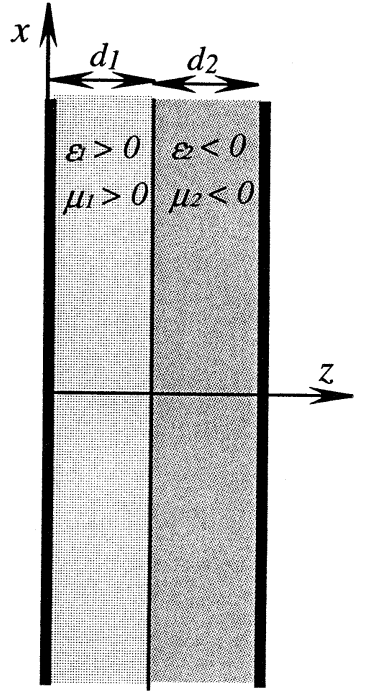

Fig. 2. An idea for a compact, subwavelength, thin cavity resonator. The two-layer structure discussed in Fig. 1 is sandwiched between the two reflectors. Our analysis shows that with the proper choice of ratio of $d_{1}$ over $d_{2}$, one can have a resonant cavity in which the ratio of $d_{1}$ and $d_{2}$ is the main constraint, not the sum of thicknesses $d_{1}+d_{2}$.

But instead it is zero!) So indeed the slab of metamaterial with $\varepsilon_{2}<0$ and $\mu_{2}<0$ at given frequencies can act as the phase compensator in this structure. This also resembles the process of phase conjugation. It is important to note that such phase cancellation in this geometry does not depend on the sum of thicknesses $d_{1}+d_{2}$; rather it depends on the ratio of $d_{1}$ and $d_{2}$. So, in principle, $d_{1}+d_{2}$ can be any value as long as $d_{1} / d_{2}$ satisfies the above condition. Therefore, even though this two-layer structure is present, the wave traversing this structure would not experience the phase difference. This feature can lead to several interesting ideas in design of some devices and components.

\section{B. Compact Subwavelength 1-D Cavity Resonators Using Metamaterials With $\varepsilon_{2}<0$ and $\mu_{2}<0$}

What we described above can be used to conceptualize an exciting possibility of designing a compact 1-D cavity resonator. We can take the above two-layer structure and put two perfect reflectors (e.g., two perfectly conducting plates) at the two open surfaces of this structure (Fig. 2). Here, we generalize the problem by assuming that the intrinsic impedances of the first layer (conventional material) and the second layer (metamaterial with $\varepsilon_{2}<0$ and $\mu_{2}<0$ at specific frequencies) are not taken to be the same as $\eta_{o}$. So here, in general, we have $\eta_{1} \neq \eta_{2} \neq \eta_{o}$ and $n_{1} \neq n_{2} \neq n_{o}$. We are now interested to solve for solutions of Maxwell equations in this cavity resonator.

\section{Formulation of the Problem}

We use the Cartesian coordinate system $(x, y, z)$, where the plane $z=0$ is taken to be at the perfectly conducting plate located at the left face of the conventional material slab shown in Fig. 2. The other perfectly conducting plate is placed at $z=$ $d_{1}+d_{2}$, which is the right face of the metamaterial slab. Since this is assumed to be a 1-D cavity resonator, all quantities are independent of the $x$ and $y$ coordinates. The time dependence for the monochromatic solutions is taken to be $\exp (-i \omega t)$. Without 
loss of generality, we take the electric and magnetic field vectors to be oriented along the $x$ and $y$ direction, respectively. In the region $0 \leq z \leq d_{1}$, where the material is a conventional lossless material, the electric and magnetic fields can be written as

$$
\begin{aligned}
& E_{x 1}=E_{o 1} \sin \left(n_{1} k_{o} z\right) \\
& H_{y 1}=\frac{n_{1} k_{o}}{i \omega \mu_{1}} E_{o 1} \cos \left(n_{1} k_{o} z\right)
\end{aligned}
$$

and in the region $d_{1} \leq z \leq d_{1}+d_{2}$, where the metamaterial with $\varepsilon_{2}<0$ and $\mu_{2}<0$ is located, the fields are written as

$$
\begin{aligned}
& E_{x 2}=E_{o 2} \sin \left[n_{2} k_{o}\left(d_{1}+d_{2}-z\right)\right] \\
& H_{y 2}=-\frac{n_{2} k_{o}}{i \omega \mu_{2}} E_{o 2} \cos \left[n_{2} k_{o}\left(d_{1}+d_{2}-z\right)\right]
\end{aligned}
$$

where subscripts " 1 " and " 2 " denote the quantities in the regions " 1 " and " 2 ," which are the conventional slab and the metamaterial slab, respectively. It is worth emphasizing that although in the above equations the values of indexes of refraction are taken to be positive quantities (and, hence, no ambiguities are introduced through the sign of the operation of square root for $\left.n_{2} \equiv \sqrt{\left(\varepsilon_{2} \mu_{2}\right) /\left(\varepsilon_{o} \mu_{o}\right)}\right)$, as will be seen shortly the choice of the sign for $n_{1}$ and $n_{2}$ will be irrelevant in the final results. The choice of the solutions presented in (1) and (2) guarantees the satisfaction of the boundary conditions at the perfectly conducting plates at $z=0$ and $z=d_{1}+d_{2}$. To satisfy the boundary conditions at the interface between the two slabs we should have

$$
\begin{aligned}
& \left.E_{x 1}\right|_{z=d_{1}}=\left.E_{x 2}\right|_{z=d_{1}} \\
& \left.H_{y 1}\right|_{z=d_{1}}=\left.H_{y 2}\right|_{z=d_{1}}
\end{aligned}
$$

which leads to

$$
\begin{aligned}
E_{o 1} \sin \left(n_{1} k_{o} d_{1}\right)-E_{o 2} \sin \left(n_{2} k_{o} d_{2}\right) & =0 \\
\frac{n_{1}}{\mu_{1}} E_{o 1} \cos \left(n_{1} k_{o} d_{1}\right)+\frac{n_{2}}{\mu_{2}} E_{o 2} \cos \left(n_{2} k_{o} d_{2}\right) & =0 .
\end{aligned}
$$

In order to have a nontrivial solution, i.e., to have $E_{o 1} \neq 0$ and $E_{o 2} \neq 0$, the determinant in (4) must vanish. That is

$$
\begin{aligned}
& \frac{n_{2}}{\mu_{2}} \sin \left(n_{1} k_{o} d_{1}\right) \cos \left(n_{2} k_{o} d_{2}\right)+\frac{n_{1}}{\mu_{1}} \sin \left(n_{2} k_{o} d_{2}\right) \cos \left(n_{1} k_{o} d_{1}\right) \\
& \quad=0
\end{aligned}
$$

which can be simplified to

$$
\frac{n_{2}}{\mu_{2}} \tan \left(n_{1} k_{o} d_{1}\right)+\frac{n_{1}}{\mu_{1}} \tan \left(n_{2} k_{o} d_{2}\right)=0 .
$$

In the above dispersion relation, the quantities $n_{1}, \mu_{1}, n_{2}, \mu_{2}$, and $k_{o}$ are all generally frequency dependent. It is important to note that the choice of sign for $n_{1}$ and $n_{2}$ does not affect this dispersion relation. Either choice of sign (positive or negative sign) for $n_{1}$ and $n_{2}$ will leave (6) unchanged. That is why we specifically mentioned earlier that we did not need to introduce any ambiguity regarding the choice of sign for $n_{2}$ in our analysis here. Since the first layer is assumed to be made of a lossless conventional material, its permeability $\mu_{1}$ is a positive real quantity. The second layer is taken to be a lossless metamaterial with $\varepsilon_{2}<0$ and $\mu_{2}<0$. Therefore, we can write $\mu_{1}=\left|\mu_{1}\right|$ and $\mu_{2}=-\left|\mu_{2}\right|$. Substituting these expressions in (6), we obtain

$$
-\frac{n_{2}}{\left|\mu_{2}\right|} \tan \left(n_{1} k_{o} d_{1}\right)+\frac{n_{1}}{\left|\mu_{1}\right|} \tan \left(n_{2} k_{o} d_{2}\right)=0 .
$$

This implies that for a given frequency $\omega$, if $\varepsilon_{1}>0, \mu_{1}>0$, $\varepsilon_{2}<0$, and $\mu_{2}<0$, a nontrivial one-dimensional (1-D) solution for this cavity is obtained when the thicknesses $d_{1}$ and $d_{2}$ satisfy the relation

$$
\frac{\tan \left(n_{1} k_{o} d_{1}\right)}{\tan \left(n_{2} k_{o} d_{2}\right)}=\frac{n_{1}\left|\mu_{2}\right|}{n_{2}\left|\mu_{1}\right|} .
$$

This relation does not show any constraint on the sum of thicknesses of $d_{1}$ and $d_{2}$. It rather deals with the ratio of tangent of these thicknesses (with multiplicative constants). So, in principle, $d_{1}$ and $d_{2}$ can conceptually be as thin or as thick as otherwise needed as long as the above ratio is satisfied. If we assume that $\omega, d_{1}$ and $d_{2}$ are chosen such that the small-argument approximation can be used for the tangent function, the above relation can be simplified as

$$
\frac{d_{1}}{d_{2}} \cong \frac{\left|\mu_{2}\right|}{\left|\mu_{1}\right|}
$$

This relation shows even more clearly how $d_{1}$ and $d_{2}$ should be related in order to have a nontrivial 1-D solution with frequency $\omega$ for this cavity. So conceptually, what is constrained here is $d_{1} / d_{2}$, not $d_{1}+d_{2}$. Therefore, in principle, one can have a thin subwavelength cavity resonator for a given frequency, if at this frequency the second layer acts a metamaterial with negative permittivity and permeability and the ratio $d_{1} / d_{2}$ satisfies the above condition. For example, for frequency of $2 \mathrm{GHz}$, if a metamaterial with negative permittivity of $-0.5 \varepsilon_{o}$ and negative permeability of $-0.5 \mu_{o}$ can be constructed as the second slab and if the conventional material slab is assumed to be air with $\varepsilon_{O}$ and $\mu_{o}$, then $n_{1}=1$ and $n_{2}=0.5$ and, thus, the required ratio of $d_{1}$ over $d_{2}$ should be $d_{1} / d_{2} \simeq 0.5$. If, in principle, this metamaterial slab can be made thin for this frequency, e.g., $d_{2}=\lambda_{o} / 10$, where $\lambda_{o}$ is the free-space wavelength of operation, then the air slab should be made with thickness $d_{1}=\lambda_{o} / 20$. Thus, the total thickness of such a thin cavity would be $d_{1}+d_{2}=3 \lambda_{o} / 20$, which for this example of 2-GHz frequency of operation would be $2.25 \mathrm{~cm}$ ! This is, of course, thinner than the conventional air cavity size of $\lambda_{o} / 2$, which would be $7.5 \mathrm{~cm}$ for $2 \mathrm{GHz}$.

The electric and magnetic field expressions for the nontrivial solutions in this 1-D cavity are given as

$$
\begin{aligned}
& E_{x 1}=E_{o} \sin \left(n_{2} k_{o} d_{2}\right) \sin \left(n_{1} k_{o} z\right) \\
& H_{y 1}=\frac{n_{1} k_{o}}{i \omega \mu_{1}} E_{o} \sin \left(n_{2} k_{o} d_{2}\right) \cos \left(n_{1} k_{o} z\right) \\
& E_{x 2}=E_{o} \sin \left(n_{1} k_{o} d_{1}\right) \sin \left[n_{2} k_{o}\left(d_{1}+d_{2}-z\right)\right] \\
& H_{y 2}=-\frac{n_{2} k_{o}}{i \omega \mu_{2}} E_{o} \sin \left(n_{1} k_{o} d_{1}\right) \cos \left[n_{2} k_{o}\left(d_{1}+d_{2}-z\right)\right]
\end{aligned}
$$

where $\mu_{1}=\left|\mu_{1}\right|$ and $\mu_{2}=-\left|\mu_{2}\right|$.

It is worth noting that if both layers 1 and 2 had been made of two conventional lossless dielectric materials, the form of the dispersion relation in (6) would have remained unchanged. 
However, both quantities $\mu_{1}$ and $\mu_{2}$ would have been positive real and, thus, if $\tan \left(n_{1} k_{o} d_{1}\right)$ had been positive, the other term $\tan \left(n_{2} k_{o} d_{2}\right)$ must have been negative in order to fulfill the dispersion relation in (6). This implies that for such a case, if $d_{1}<$ $\pi / 2 n_{1} k_{o}$ for $\tan \left(n_{1} k_{o} d_{1}\right)$ to be positive, then $d_{2}$ must have been greater than $\pi / 2 n_{2} k_{o}$ in order to have $\tan \left(n_{2} k_{o} d_{2}\right)$ negative, which puts some limits on the sum of thicknesses $d_{1}$ and $d_{2}$. In the case under study in this letter, however, since $\mu_{1}>0$ and $\mu_{2}<0$, the two functions $\tan \left(n_{1} k_{o} d_{1}\right)$ and $\tan \left(n_{2} k_{o} d_{2}\right)$ can be both positive (or both negative) and, as a result, there is no constraint on the sum of $d_{1}$ and $d_{2}$.

Finally, one notes that the electric field expressions may possess discontinuous first derivatives (with respect to $z$ ) at the boundary between the two layers, i.e.,

$$
\left.\frac{\partial E_{x 1}}{\partial z}\right|_{z=d_{1}} \neq\left.\frac{\partial E_{x 2}}{\partial z}\right|_{z=d_{1}} .
$$

This should not cause any concern since according to the Maxwell equations, the tangential component of the magnetic field in this problem, which is expressed as $H_{y}=\frac{1}{i \omega \mu} \frac{\partial E_{x}}{\partial z}$ are continuous at such a boundary. This implies that $\frac{1}{i \omega \mu_{1}} \frac{\partial E_{x 1}}{\partial z}=\frac{1}{i \omega \mu_{2}} \frac{\partial E_{x 2}}{\partial z}$. Since in our problem here, $\mu_{1}>0$ and $\mu_{2}<0$, the first $z$-derivatives of electric field $\partial E_{x 1} / \partial z$ and $\partial E_{x 2} / \partial z$ have opposite signs at the interface between the two layers.

\section{SUMMARY}

We have introduced ideas for 1-D cavity resonators utilizing the concept of lossless metamaterials in which both permittivity and permeability possess negative real values at given frequencies. We have shown that a slab of metamaterial having negative permittivity and permeability can function as a phase compensator/conjugator. Our analysis has also shown that when the cavity is filled with two layers of materials; the first layer assumed to be a lossless conventional material and the second layer is taken to be the metamaterial with negative permittivity and permittivity, the nontrivial 1-D solutions for such a cavity, in principle, depend on the ratio of thicknesses of the two layers, not the sum of thicknesses. In other words, the cavity can conceptually be thin and can still be resonant, as long as the ratio of thicknesses is satisfied in the special dispersion relation. This can, in principle, provide possibility for having subwavelength thin compact cavity resonators. Such subwavelength cavity resonators can lead to very interesting designs for various compact subwavelength devices and components.

\section{REFERENCES}

[1] W. E. Kock, "Metallic delay lenses," Bell Syst. Tech. J., vol. 27, pp. $58-82,1948$.

[2] J. Brown, "Artificial dielectrics," in Progress in Dielectrics, J. B. Birks and J. H. Schulman, Eds. London, U.K.: Heywood, 1960, vol. 2, pp. 193-225.

[3] D. L. Jaggard, A. R. Mickelson, and C. H. Papas, "On electromagnetic waves in chiral media," J. Appl. Phys., vol. 18, pp. 211-216, 1979.
[4] I. P. Theron and J. H. Cloete, "The optical activity of an artificial nonmagnetic uniaxial chiral crystal at microwave frequencies," J. Electromagn. Waves Applicat., vol. 10, pp. 539-561, 1996.

[5] M. M. I. Saadoun and N. Engheta, "A reciprocal phase shifter using novel pseudochiral or $\Omega$ medium," Microwave Opt. Technol. Lett., vol. 5, no. 4, pp. 184-188, 1992.

[6] C. A. Moses and N. Engheta, "An idea for electromagnetic "feedforward-feedbackward' media," IEEE Trans. Antennas Propagation, vol. 47, no. 5, pp. 918-928, 1999.

[7] I. V. Lindell, A. H. Sihvola, S. A. Tretyakov, and A. J. Viitanen, Electromagnetic Waves in Chiral and Bi-Isotropic Media. Boston, MA: Artech House, 1994.

[8] K. W. Whites, "Full-wave computation of constitutive parameters for lossless composite chiral materials," IEEE Trans. Antennas Propagat., vol. 43, pp. 376-384, 1995.

[9] A. L. Topa, C. R. Paiva, and A. M. Barbosa, "Radiation control on a step discontinuity of a grounded chiral slab," in Proc. Bianisotropics, Lisbon, Portugal, Sept. 2000, pp. 325-328.

[10] L. R. Arnaut, "Interaction between bianisotropic particles," J. Electromagn. Waves Applicat., vol. 11, pp. 133-137, 1997.

[11] A. Lakhtakia, "Isotropic Maxwell-Garnett model for biisotropic-in-biisotropic mixtures," Int. J. Infrared Millimeter Waves, vol. 13, no. 4, pp. 551-558, 1992.

[12] W. S. Weiglhofer, A. Lakhtakia, and C. J. Monzon, "Maxwell-Garnett model for composites of electrically small uniaxial objects," Microwave Opt. Technol. Lett., vol. 6, no. 12, pp. 681-684, 1993.

[13] A. H. Sihvola, Electromagnetic Mixing Formulas and Applications. London, U.K.: Inst. Elect. Eng., 1999.

[14] G. Kristensson, S. Rikte, and A. H. Sihvola, "Mixing formulas in the time domain," J. Opt. Soc. Amer. A., vol. 15, no. 5, pp. 1411-1422, 1998.

[15] I. V. Lindell and F. Olyslager, "Potentials in bi-anisotropic media," $J$. Electromagn. Waves Applicat., vol. 15, no. 1, pp. 3-18, 2001.

[16] F. Auzanneau and R. W. Ziolkowski, "Theoretical study of synthetic bianisotropic materials," J. Electromagn. Waves Applicat., vol. 12, p. 353, 1998.

[17] D. R. Smith, W. J. Padilla, D. C. Vier, S. C. Nemat-Nasser, and S. Schultz, "Composite medium with simultaneously negative permeability and permittivity," Phys. Rev. Lett., vol. 84, no. 18, pp. 4184-4187, May 2000.

[18] D. R. Smith and N. Kroll, "Negative refractive index in left-handed materials," Phys. Rev. Lett., vol. 85, no. 14, pp. 2933-2936, Oct. 2000.

[19] J. B. Pendry, "Negative refraction makes a perfect lens," Phys. Rev. Lett., vol. 85 , no. 18 , pp. $3966-3969$, Oct. 2000

[20] R. A. Shelby, D. R. Smith, S. C. Nemat-Nasser, and S. Schultz, "Microwave transmission through a two-dimensional, isotropic, left-handed metamaterial," Appl. Phys. Lett., vol. 78, no. 4, pp. 489-491, Jan. 2001.

[21] R. A. Shelby, D. R. Smith, and S. Schultz, "Experimental verification of a negative index of refraction," Sci., vol. 292, no. 5514, pp. 77-79, Apr. 2001.

[22] V. G. Veselago, "The electrodynamics of substances with simultaneously negative values of $\varepsilon$ and $\mu$ " (in also in Russian, Usp. Fiz. Nauk., vol. 92, pp. 517-526, 1967), Soviet Phys. Uspekhi, vol. 10, no. 4, pp. $509-514,1968$

[23] M. M. I. Saadoun and N. Engheta, "Theoretical study of electromagnetic properties of nonlocal omega media," in Progress in Electromagnetic Research (PIER) Monograph Series: Bianisotropic and Bi-Isotropic Media and Applications, A. Priou, Ed. Cambridge, MA: EMW, 1994, vol. 9, ch. 15, pp. 351-397.

[24] I. V. Lindell, S. A. Tretyakov, K. I. Nikoskinen, and S. Ilvonen, "BW media-Media with negative parameters, capable of supporting backward waves," Helsinki Univ. Technol., 366, ser. Electromagn. Lab. Rep. Ser., Apr. 2001.

[25] R. W. Ziolkowski, "Superluminal transmission of information through an electromagnetic metamaterials," Phys. Rev. E., vol. 63, no. 4, 046 604, Apr. 2001.

[26] R. W. Ziolkowski and E. Heyman, "Wave propagation in media having negative permittivity and permeability," Phys. Rev. E., vol. 64, no. 5, 056625 , Oct. 2001

[27] N. Engheta, "Compact cavity resonators using metamaterials with negative permittivity and permeability," in Proc. Int. Conf. Electromagnetics in Advanced Applications (ICEAA), Torino, Italy, Sept. 2001, pp. 739-742. 